\title{
EFEITO DA TEMPERATURA NO DESEMPENHO CATALÍTICO DE ÓXIDOS DE FERRO CONTENDO COBRE E ALUMÍNIO
}

Genira Carneiro de Araujo, Alexilda Oliveira de Souza e Maria do Carmo Rangel*

Instituto de Química, Universidade Federal da Bahia, Campus Universitário de Ondina, 40170-290 Salvador - BA

Eduardo Antônio Pinheiro

Departamento de Química, Universidade Federal de Santa Catarina, Campus Universitário, Trindade, 88040-900 Florianópolis - SC

Recebido em 28/7/00; aceito em 22/8/01

THE EFFECT OF TEMPERATURE ON THE CATALYTIC PERFORMANCE OF IRON OXIDE WITH COPPER AND ALUMINUM. Aluminum and copper doped hematite was evaluated in the high temperature shift (HTS) reaction at several temperatures in order to find catalysts that can work in different operational conditions. It was found that the catalysts work in kinetic regime in the range of $300-400{ }^{\circ} \mathrm{C}$. Both copper and aluminum increases the activity and selectivity. Aluminum acts as textural promoter whereas copper acts as structural one. The most promising catalyst is that with both copper and aluminum which showed higher activity and selectivity than a commercial sample. This catalyst has the advantages of being non toxic and can work at low temperatures.

Keywords: hydrogen production; iron oxides; CO conversion.

\section{INTRODUÇÃO}

A reforma de hidrocarbonetos, em presença de vapor, é a principal via de obtenção de hidrogênio de alta pureza, para diversas aplicações industriais, tais como síntese da amônia, reações de hidrogenação e reforma catalítica de nafta de petróleo ${ }^{1}$. Esse processo envolve diversas etapas, incluindo a conversão de monóxido a dióxido de carbono com vapor d'água, uma reação reversível e moderadamente exotérmica ${ }^{2-4}$ :

$$
\mathrm{CO}_{(\mathrm{g})}+\mathrm{H}_{2} \mathrm{O}_{(\mathrm{g})} \rightleftarrows \mathrm{CO}_{2(\mathrm{~g})}+\mathrm{H}_{2(\mathrm{~g})} \quad \Delta \mathrm{H}=-9,84 \text { kcal.mol }^{-1}
$$

Em plantas industriais, essa reação remove o monóxido de carbono residual, em correntes gasosas provenientes da reforma do gás natural ou nafta de petróleo, que atua como veneno para o catalisador de síntese de amônia e diversos catalisadores metálicos, usados na hidrogenação ${ }^{1,5}$. Além disso, a reação contribui para o aumento da produção de hidrogênio ${ }^{3.4}$.

Para se obter conversões viáveis do ponto de vista econômico, a reação é conduzida em duas etapas, em plantas industriais. A primeira ocorre na faixa de $350-420^{\circ} \mathrm{C}$, sob condições cinéticas favoráveis e é conhecida como reação de HTS (High Temperature Shift), enquanto a outra - denominada reação de LTS (Low Temperature Shift) - ocorre em temperaturas próximas a $200{ }^{\circ} \mathrm{C}^{4,5}$.

Em processos comerciais, a reação de HTS é conduzida sobre um leito catalítico de óxido de ferro contendo óxido de cromo, que atua como promotor textural, evitando a sinterização do catalisador, ao longo da sua vida útil ${ }^{2,5-8}$. Recentemente, o cobre foi incluído na formulação desse sólido produzindo sistemas ainda mais ativos e seletivos $^{9-12}$.

Os catalisadores comerciais possuem uma série de vantagens como baixo custo, estabilidade térmica e resistência a venenos ${ }^{5,13}$. São comercializados na forma de hematita $\left(\alpha-\mathrm{Fe}_{2} \mathrm{O}_{3}\right)$ e são reduzidos in situ para dar origem à magnetita $\left(\mathrm{Fe}_{3} \mathrm{O}_{4}\right)$, que é a fase ativa.

\footnotetext{
*e-mail: mcarmov@ufba.br
}

No processo de redução, em plantas de amônia, utiliza-se normalmente o gás de processo constituído por uma mistura de cerca de $10 \% \mathrm{CO}, 10 \% \mathrm{CO}_{2}, 60 \% \mathrm{H}_{2}$ e $20 \% \mathrm{~N}_{2}$, além de traços de metano e argônio, oriunda da reforma a vapor de gás natural ou de nafta de petróleo ${ }^{3}$. A reação de redução é altamente exotérmica e deve ser controlada, de modo a não danificar o reator ou o catalisador ${ }^{3-5}$. Em especial, deve-se evitar a formação de ferro metálico, que pode catalisar reações indesejáveis, como a produção de hidrocarbonetos, levando a uma diminuição da seletividade e à desativação do catalisador $^{4,5}$. Em processos industriais, a redução é controlada pela injeção de grandes quantidades de vapor d'água ao sistema reacional que, todavia, leva a um aumento dos custos de operação ${ }^{3}$.

Em virtude da demanda por tecnologias cada vez mais flexíveis, que permitam processar cargas de diversas procedências, a diferentes temperaturas, existe o interesse pelo desenvolvimento de catalisadores que possam operar, com eficiência, em diferentes condições de operação. Em particular, é desejável a operação a temperaturas baixas, com a finalidade de reduzir os custos de energia; além disso, esse procedimento pode aumentar a vida útil do catalisador, pela diminuição da severidade da operação. Como se processam milhares de toneladas por ano, uma redução de alguns graus de temperatura pode representar uma diminuição significativa dos custos de operação.

Em estudos anteriores ${ }^{11,12}$, verificou-se que o alumínio é um dopante promissor para substituir o cromo em catalisadores de HTS à base de óxidos de ferro contendo cobre. No presente trabalho, óxidos de ferro, na forma de hematita e contendo alumínio e cobre, foram testados em diferentes temperaturas, visando avaliar a flexibilidade de operação desses sistemas. Enquanto os testes a baixas temperaturas irão fornecer informações sobre a possibilidade do catalisador operar em condições mais econômicas, o seu desempenho a altas temperaturas irá refletir a sua resistência à desativação.

\section{PARTE EXPERIMENTAL}

Os materiais foram preparados através do processo sol-gel, dissolvendo-se $0,25 \mathrm{~mol}$ de nitrato de ferro III $\left[\mathrm{Fe}\left(\mathrm{NO}_{3}\right)_{3} .9 \mathrm{H}_{2} \mathrm{O}\right]$ e 0,02 
mol de nitrato de alumínio $\left[\mathrm{Al}\left(\mathrm{NO}_{3}\right)_{3} \cdot 9 \mathrm{H}_{2} \mathrm{O}\right]$ em $250 \mathrm{~mL}$ de água. Esta solução foi adicionada, por meio de uma bomba peristáltica, a um béquer contendo $50 \mathrm{~mL}$ de água, simultaneamente com uma solução de hidróxido de amônio p.a., sob agitação magnética e à temperatura ambiente. O sistema permaneceu sob agitação durante $30 \mathrm{~min}$ e, em seguida, foi centrifugado (a $2000 \mathrm{rpm}$ e $10 \mathrm{~min}$ ). O gel obtido foi lavado quatro vezes, com $100 \mathrm{~mL}$ de água deionizada a $60{ }^{\circ} \mathrm{C}$, e depois impregnado com uma solução de nitrato de cobre preparada por dissolução de $0,02 \mathrm{~mol} \mathrm{de} \mathrm{Cu}\left(\mathrm{NO}_{3}\right)_{2} .3 \mathrm{H}_{2} \mathrm{O} \mathrm{em}$ $250 \mathrm{~mL}$ de água deionizada, por $24 \mathrm{~h}$. Em seguida foi centrifugado, seco em estufa a $120{ }^{\circ} \mathrm{C}$ por $12 \mathrm{~h}$, triturado e peneirado em 100 mesh. O sólido foi, então, calcinado a $500{ }^{\circ} \mathrm{C}$, por $2 \mathrm{~h}$, sob fluxo de nitrogênio (100 mL/min), obtendo-se a Amostra HAC.

Foram obtidos também materiais contendo apenas alumínio (Amostra $\mathrm{HA}$ ), cobre (Amostra $\mathrm{HC}$ ), além da hematita pura (Amostra $\mathrm{H}$ ).

$\mathrm{O}$ teor dos metais nos catalisadores foi determinado dissolvendose $0,1 \mathrm{~g}$ do sólido em ácido clorídrico concentrado e mantendo-se o sistema sob refluxo. O líquido obtido foi levado até quase à secura e, então, avolumado a $100 \mathrm{~mL}$ com solução de ácido clorídrico $1 \%$. As amostras foram analisadas num espectrômetro de emissão atômica em plasma indutivamente acoplado (ICP/AES) Arl modelo 3410.

Os difratogramas de raios $\mathrm{X}$ foram obtidos por meio de um equipamento Shimadzu, modelo XD3A, usando filtro de níquel e radiação de $\mathrm{CuK} \propto$, gerada a $35 \mathrm{kV}$ e $25 \mathrm{~mA}$. Os experimentos de microscopia eletrônica de varredura foram conduzidos com amostras selecionadas, em um microscópio Jeol modelo JSM-T300, operando a 20-30 KV. As microanálises de raios X foram efetuadas usando uma microssonda Noran, acoplada a esse microscópio.

As áreas específicas dos catalisadores foram medidas usando-se um equipamento Micromeritics modelo TPD/TPR 2900 Analyser e o método BET. Utilizou-se uma massa de cerca de $0,20 \mathrm{~g}$ da amostra, previamente aquecida em atmosfera de nitrogênio a $150{ }^{\circ} \mathrm{C}$, por $1 \mathrm{~h}$. As medidas foram conduzidas com uma mistura $30 \% \mathrm{~N}_{2} / \mathrm{He}$, à temperatura do nitrogênio líquido (BET).

Através de calorimetria diferencial de varredura (DSC) e termogravimetria (TG), em atmosfera inerte (usando os precursores) e redutora (usando os catalisadores), acompanhou-se a formação da hematita e da magnetita respectivamente. As medidas foram conduzidas num aparelho Schimadzu, modelo 50, sob fluxo de nitrogênio ou de uma mistura $50 \% \mathrm{H}_{2} / \mathrm{N}_{2}(100 \mathrm{~mL} / \mathrm{min})$. As amostras foram aquecidas da temperatura ambiente até $500{ }^{\circ} \mathrm{C}$ a uma velocidade de 10 graus/min.

$\mathrm{O}$ desempenho dos catalisadores produzidos, em relação à reação de HTS, foi avaliado em um teste microcatalítico, constituído de um reator de leito fixo, operando a 1 atm e diferentes temperaturas de reação $\left(250,300,350,370,400{ }^{\circ} \mathrm{C}\right)$, utilizando-se uma vazão de alimentação de $40 \mathrm{~mL} / \mathrm{min}$ e razão molar vapor/gás de processo igual a 0,6. O arranjo experimental está descrito em trabalho anterior ${ }^{9}$.

Após esses experimentos, analisou-se a razão $\mathrm{Fe}(\mathrm{II}) / \mathrm{Fe}$ (III) dos catalisadores, para verificar a sua estabilidade no meio reacional. Os teores de $\mathrm{Fe}$ (II) foram determinados dissolvendo-se as amostras numa solução de ácido clorídrico $18 \%$, sob refluxo, e em atmosfera de dióxido de carbono, seguida da titulação com dicromato de potássio, usando difenilaminosulfonato de bário como indicador ${ }^{14}$. Os teores de ferro total foram obtidos como descrito anteriormente e os de $\mathrm{Fe}$ (III) foram obtidos por diferença.

\section{RESULTADOS E DISCUSSÃO}

A Tabela 1 mostra os resultados de análise química de ferro, alumínio e cobre. Observa-se que a razão Fe/dopante nos catalisadores é próxima à da solução de partida, indicando que as condições usadas foram favoráveis à precipitação de compostos de ferro e de alumínio e que a adsorção de cobre, pelas partículas de hidróxidos de ferro, não foi afetada pelo alumínio. Os hidróxidos ou óxidos hidratados de ferro ou alumínio são descritos na literatura como precipitados gelatinosos, com elevadas áreas específicas e alta capacidade de adsorção ${ }^{15,16}$. Como consequência, esses materiais sorvem facilmente íons presentes ou adicionados à solução ${ }^{17}$. Dessa forma, é de se esperar que os íons sejam adsorvidos pelo gel durante a etapa de impregnação, nas amostras do presente trabalho.

Observou-se a presença da hematita nos catalisadores novos, em concordância com estudos anteriores ${ }^{5,9,12}$. A Figura 1 ilustra o padrão de difração obtido. Não se observou a formação de nenhuma outra fase e este fato pode ser explicado pela similaridade dos raios iônicos das espécies $\mathrm{Cu}^{2+}, \mathrm{Al}^{3+} \mathrm{e} \mathrm{Fe}^{3+}$, que facilitam a incorporação dos dois primeiros na rede cristalina do óxido de ferro ${ }^{18,19}$.

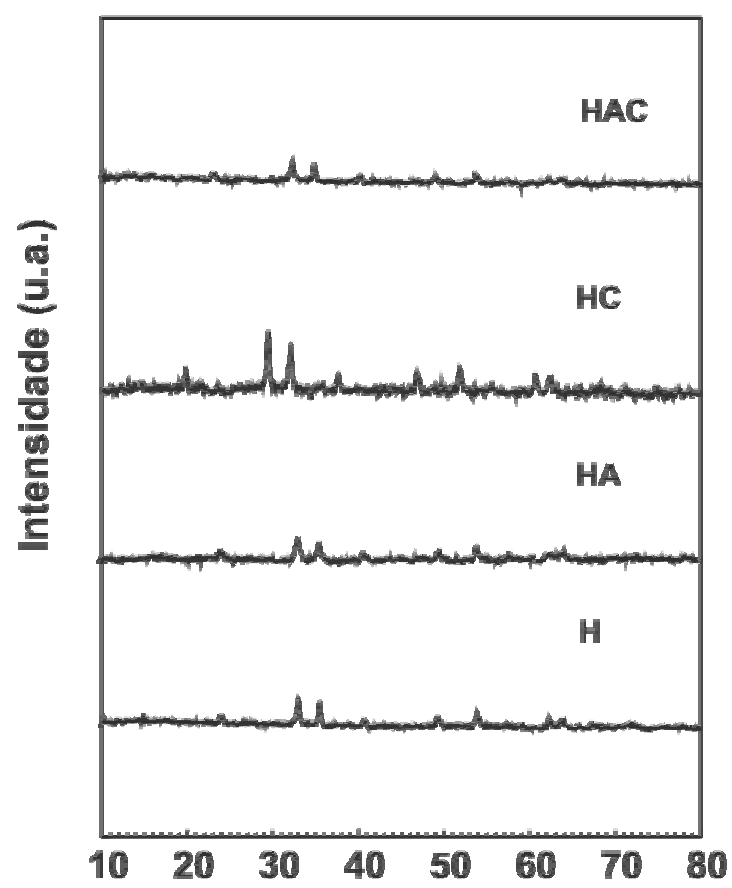

$2 \theta$ (graus)

Figura 1. Difratogramas de raios $X$ dos catalisadores sem dopante (Amostra $H$ ), contendo alumínio (HA), cobre (Amostra HC) e os dois dopantes (HAC)

Tabela 1. Resultados de análise química dos catalisadores obtidos. Amostra $\mathrm{H}$ : hematita; Amostras $\mathrm{HA}$ e $\mathrm{HC}$ : com $\mathrm{Fe} / \mathrm{Al}=10$ e $\mathrm{Fe} / \mathrm{Cu}=10$ respectivamente; Amostra $\mathrm{HAC}$ : com $\mathrm{Fe} / \mathrm{Al}=10$ e $\mathrm{Fe} / \mathrm{Cu}=10$

\begin{tabular}{cccccc}
\hline Amostra & $\% \mathrm{Fe}( \pm 0,05)$ & $\% \mathrm{Al}( \pm 0,03)$ & $\% \mathrm{Cu}( \pm 0,03)$ & $\mathrm{Fe} / \mathrm{Al}( \pm 0,05)$ & $\mathrm{Fe} / \mathrm{Cu}( \pm 0,05)$ \\
\hline H & 70,66 & - & - & - & - \\
HA & 66,18 & 3,22 & - & - & - \\
HC & 61,36 & - & 7,76 & 9,96 & 9,00 \\
HAC & 57,24 & 2,84 & 7,88 & 9,00 \\
\hline
\end{tabular}


Os experimentos de análise térmica obtidos em atmosfera inerte (nitrogênio), e usando os precursores dos catalisadores (Figura 2), foram conduzidos com a finalidade de acompanhar a formação do catalisador (hematita). As curvas de DSC apresentaram picos endotérmicos em temperaturas inferiores a $200{ }^{\circ} \mathrm{C}$, que podem ser atribuídos à perda de material voláti ${ }^{20,21}$. Os termogramas mostraram ainda um pico exotérmico em torno de $250^{\circ} \mathrm{C}$ e outro na faixa de 470 a $550{ }^{\circ} \mathrm{C}$. O primeiro se deve provavelmente à formação de um óxido de ferro hidratado não-cristalino e é acompanhado de perda de massa, na faixa de $300 \mathrm{a} 450^{\circ} \mathrm{C}$, que pode ser devido à desidratação desse composto ${ }^{22}$. O outro pico é característico da formação de hematita ${ }^{23}$, em concordância com os resultados de difração de raiosX. A partir da Figura 2, observa-se que este pico é deslocado para temperaturas mais altas devido à presença do cobre, indicando que esse dopante dificulta a formação do óxido. O mesmo ocorre com as curvas dos materiais contendo alumínio ou os dois dopantes.

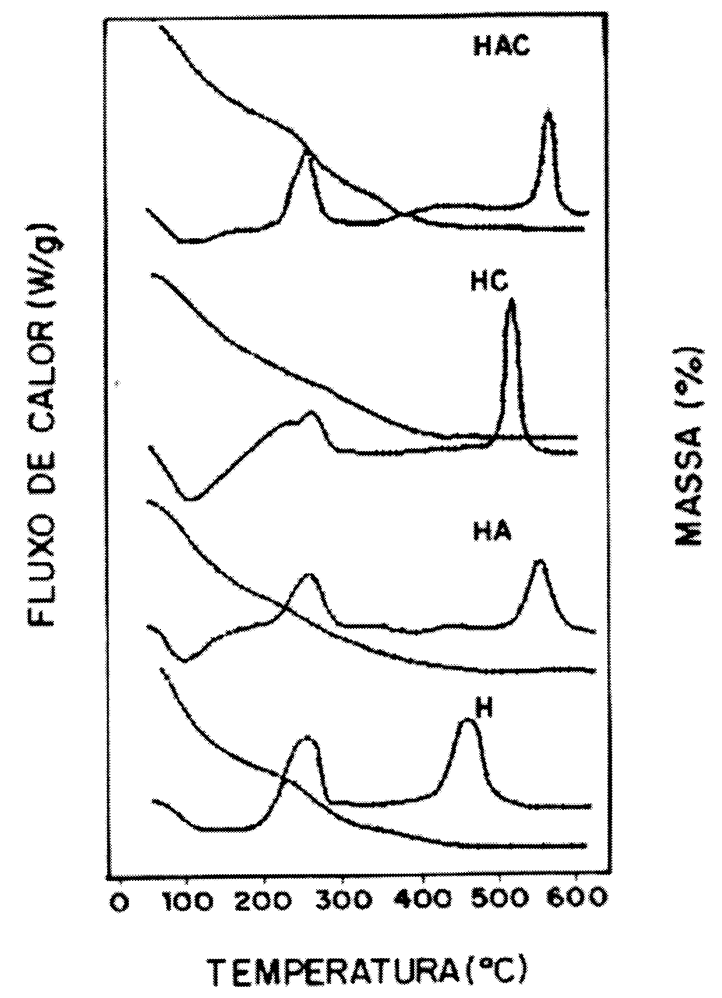

Figura 2. Curvas de calorimetria diferencial de varredura e análise termogravimétrica dos precursores dos catalisadores, obtidos em atmosfera inerte. Amostra H: sem dopante; Amostras HA e HC: com alumínio e cobre respectivamente; Amostra HAC: com alumínio e cobre

As áreas específicas dos catalisadores aumentaram devido à presença do alumínio e não foram alteradas pelo cobre. A presença simultânea dos dois dopantes levou a um aumento significativo da área, em relação àquela contendo alumínio, sendo superior à de uma amostra comercial $\left(50 \mathrm{~m}^{2} \cdot \mathrm{g}^{-1}\right)$. Os resultados são mostrados na Tabela 2.

Tabela 2. Área específica $(\mathrm{Sg})$ da hematita pura $(\mathrm{H})$, contendo alumínio (HA), cobre (HC) e alumínio e cobre (HAC)

\begin{tabular}{cc}
\hline Amostra & $\mathrm{S}_{\mathrm{g}}\left(\mathrm{m}^{2} \cdot \mathrm{g}^{-1}\right)$ \\
\hline H & 27 \\
HA & 59 \\
HC & 26 \\
HAC & 86 \\
\hline
\end{tabular}

A maior resistência à sinterização, apresentada pelos materiais contendo alumínio, está em concordância com trabalhos anteriores, em que se observou que esse metal atua como promotor textural ${ }^{11,12}$. Essa ação tem sido tradicionalmente associada com um fenômeno no qual o alumínio atua, na superfície do sólido, separando as partículas de óxido de ferro ${ }^{3}$. Entretanto, Topsoe et al. ${ }^{24}$ mostraram que, em catalisadores de síntese de amônia, uma grande fração do alumínio permanece no interior das partículas de óxidos de ferro, como uma fase ocluída, causando tensões na rede e deslocando o equilíbrio para a formação de partículas menores, já que a razão tensão/ superfície se torna menor para partículas menores. Dessa forma o alumínio, tanto na superfície como no interior do sólido, pode contribuir para diminuir a sinterização do material.

Os resultados obtidos por microscopia eletrônica de varredura mostraram partículas com diferentes formas e tamanhos, com vários defeitos e imperfeições, que podem ser atribuídos aos numerosos fatores, que afetam as reações envolvidas na precipitação dos compostos e que são difíceis de controlar ${ }^{25}$. Os experimentos com microssonda eletrônica de raios-X mostraram uma distribuição irregular dos promotores, nas camadas mais próximas à superfície.

Os termogramas obtidos em atmosfera redutora mostraram um pico exotérmico a temperaturas inferiores a $250{ }^{\circ} \mathrm{C}$, devido à formação de magnetita, e um endotérmico a temperaturas mais altas atribuído à produção de ferro metálico ${ }^{26}$. A Tabela 3 mostra os valores obtidos com as diversas amostras. Observa-se que a presença do cobre desloca o pico, associado à magnetita, para temperaturas mais baixas, indicando que esse metal facilita a formação dessa fase. Por outro lado, o efeito térmico associado à produção de ferro metálico ocorre a temperaturas mais altas $\left(430{ }^{\circ} \mathrm{C}\right)$ que no caso da hematita $\left(300{ }^{\circ} \mathrm{C}\right)$, devido à presença de cobre, indicando que esse metal dificulta o processo de redução da magnetita. O termograma da amostra contendo apenas alumínio não apresentou picos na faixa de temperatura estudada, indicando a inexistência dos processos de redução e, portanto, o efeito desse metal em inibi-los. Todavia, não se pode descartar a possibilidade da ocorrência simultânea de processos endotérmicos e exotérmicos, inviabilizando sua detecção por $\operatorname{DSC}^{27}$. A curva do material contendo os dois dopantes mostrou o pico devido à formação da magnetita deslocado para temperaturas mais baixas, indicando que a presença simultânea do cobre e alumínio favorece ainda mais a redução da hematita à magnetita, em relação à amostra isenta de metais. Por outro lado, a curva da Amostra HAC não apresentou picos endotérmicos a altas temperaturas, indicando que os metais dopantes evitam a redução da magnetita a ferro metálico, nessa faixa de temperatura. Dessa forma, pode-se concluir que a presença do cobre e alumínio aumenta a resistência do catalisador à desativação por destruição da fase ativa (magnetita) para formar ferro metálico.

Tabela 3. Temperaturas iniciais de formação da magnetita (Tm) e do ferro metálico (Tf) dos catalisadores obtidos. Amostra H: hematita; Amostras $\mathrm{HA}$ e $\mathrm{HC}$ : com $\mathrm{Fe} / \mathrm{Al}=10$ e $\mathrm{Fe} / \mathrm{Cu}=10$ respectivamente; Amostra HAC: $c 0 m \mathrm{Fe} / \mathrm{Al}=10$ e $\mathrm{Fe} / \mathrm{Cu}=10$

\begin{tabular}{ccc}
\hline Amostra & $\operatorname{Tm}\left({ }^{\circ} \mathrm{C}\right)$ & Tf $\left({ }^{\circ} \mathrm{C}\right)$ \\
\hline H & 200 & 300 \\
HÁ & - & - \\
HC & 190 & 430 \\
HAC & 150 & -
\end{tabular}

Os resultados da avaliação catalítica (Tabela 4) mostraram que, de modo geral, os sólidos se tornaram mais ativos a temperaturas mais altas, o que pode ser atribuído a fatores cinéticos. Nesta faixa de temperatura, as constantes de equilíbrio são baixas e é geralmente aceito 
Tabela 4. Atividade catalítica (a), seletividade (\%S) e razão Fe(II)/Fe(III) após os testes catalíticos de catalisadores à base de hematita (H), hematita dopada com alumínio (HA), hematita com cobre (HC) e hematita dopada com alumínio e cobre (HAC)

\begin{tabular}{|c|c|c|c|c|c|c|c|c|c|c|}
\hline \multirow[t]{2}{*}{ Amostra } & \multicolumn{5}{|c|}{ a x $10^{4}\left(\mathrm{~mol} \cdot \mathrm{g}^{-1} \cdot \mathrm{h}^{-1}\right)$} & \multirow[b]{2}{*}{$250^{\circ} \mathrm{C}$} & \multirow[b]{2}{*}{$300^{\circ} \mathrm{C}$} & \multicolumn{3}{|c|}{ Seletividade $(\%)$} \\
\hline & $250^{\circ} \mathrm{C}$ & $300^{\circ} \mathrm{C}$ & $350^{\circ} \mathrm{C}$ & $370^{\circ} \mathrm{C}$ & $400^{\circ} \mathrm{C}$ & & & $350^{\circ} \mathrm{C}$ & $370^{\circ} \mathrm{C}$ & $400^{\circ} \mathrm{C}$ \\
\hline $\mathrm{H}$ & 1 & 3 & 5 & 7 & 13 & 3 & 6 & 10 & 50 & 19 \\
\hline HA & 0 & 0 & 6 & 9 & 11 & - & - & 5 & 10 & 18 \\
\hline $\mathrm{HC}$ & 6 & 10 & 24 & 24 & 25 & 20 & 27 & 57 & 80 & 53 \\
\hline HAC & 21 & 43 & 30 & 34 & 38 & 25 & 31 & 82 & 70 & 63 \\
\hline
\end{tabular}

que, nestas condições, o sistema opera sob regime cinético ${ }^{4}$. Esse comportamento também indica que os catalisadores são estáveis nessa faixa de temperatura. Entretanto, a amostra contendo apenas cobre levou à mesma conversão em todas as temperaturas, sugerindo que ocorre desativação do catalisador, a temperaturas elevadas, compensando o aumento da atividade por efeito da temperatura. É conhecido que o cobre sofre sinterização a temperaturas superiores a $300{ }^{\circ} \mathrm{C}$, o que justifica os resultados obtidos ${ }^{4}$. Em todas as temperaturas, a atividade aumentou devido à presença do cobre, o que pode ser atribuído a uma modificação na natureza dos sítios de ferro, assim como à atuação catalítica do cobre. É conhecido que esse metal é cataliticamente ativo para a reação de $\mathrm{HTS}^{4}$. A presença simultânea dos dois dopantes melhorou o desempenho catalítico dos sólidos, devido à ação textural do alumínio e ao efeito estrutural do cobre ${ }^{12}$.

A atividade obtida com uma mistura mecânica $\left(3 \times 10^{-4} \mathrm{~mol} \cdot \mathrm{g}^{-1} \cdot \mathrm{h}^{-1}\right)$, do catalisador contendo alumínio e óxido de cobre, foi mais baixa que a da Amostra HAC $\left(7 \times 10^{-4} \mathrm{~mol} \cdot \mathrm{g}^{-1} \cdot \mathrm{h}^{-1}\right)$ medida a $200{ }^{\circ} \mathrm{C}$. Esse resultado indica que o aumento da atividade catalítica não pode ser atribuída apenas a um aumento do número de sítios ativos do cobre, que se encontra presente em baixa concentração. Dessa forma, podese admitir que a ação promotora do cobre está relacionada às interações entre os metais (cobre e ferro), causando modificações eletrônicas nos átomos de ferro. A influência dessas modificações eletrônicas na atividade dos catalisadores dopados com cobre pode ser explicada através dos dois mecanismos descritos na literatura. No mecanismo regenerativo, a superfície passa por sucessivos ciclos de redução e oxidação pela água e pelo monóxido de carbono, respectivamente, para formar os correspondentes produtos hidrogênio e monóxido de carbono ${ }^{28}$. As condições para a ocorrência desses ciclos são mais favoráveis nos sólidos dopados com cobre, como mostrado pelos resultados de DSC, conduzidos sob atmosfera redutora, a partir dos quais se conclui que o cobre favorece a formação da magnetita. No mecanismo adsortivo, os reagentes são adsorvidos pela superfície do catalisador, onde reagem para formar compostos superficiais intermediários, tais como formiatos, seguido pela decomposição a produtos e dessorção da superfície ${ }^{29}$. A maior mobilidade dos átomos de oxigênio e de espécies hidroxila da rede nos catalisadores contendo cobre, devido a um aumento dos elétrons de valência do cobre quando comparados aos do ferro, leva a um aumento da condutividade e da atividade catalítica.

Os resultados obtidos mostraram que os catalisadores estudados podem ser usados a $400{ }^{\circ} \mathrm{C}$, sem sofrer perdas nas suas propriedades. Devido à alta atividade desses materiais, conclui-se também que eles podem ser usados em temperaturas mais baixas, estabelecendo-se o valor mínimo em que eles apresentem desempenho próximo ao catalisador usado na reação de LTS. É conhecido que os catalisadores de LTS sofrem acentuadas perdas de atividade aumentando, de forma significativa, os custos de produção $0^{4}$. Pode-se reduzir esses custos, conduzindo-se o processo em uma única etapa, eliminando-se a reação de LTS. A amostra mais promissora, para uma aplicação industrial, é aquela contendo cobre e alumínio, que apresenta ativida- de muito superior ao catalisador comercial contendo cromo e cobre $\left(25 \times 10^{-4} \mathrm{~mol} \cdot \mathrm{g}^{-1} \cdot \mathrm{h}^{-1}\right)$, podendo ser empregada em processos conduzidos a temperaturas inferiores $\left(350^{\circ} \mathrm{C}\right)$ àquelas empregadas em plantas industriais $\left(370^{\circ} \mathrm{C}\right)$. Os valores da razão $\mathrm{Fe}(\mathrm{II}) / \mathrm{Fe}(\mathrm{III})$ foram iguais ao valor estequiométrico da magnetita, indicando que os catalisadores são estáveis sob as condições da reação.

Os valores de seletividade referentes à produção de dióxido de carbono indicaram que esse parâmetro aumentou, de modo significativo, devido à presença simultânea do cobre e do alumínio, em todas as temperaturas, como mostram os dados da Tabela 4. De fato, a Amostra HAC apresentou seletividade na faixa de 25 a $63 \%$, enquanto a hematita pura mostrou valores entre 3 e $19 \%$. O catalisador mais ativo (HAC) foi mais seletivo do que uma amostra comercial $(\mathrm{S}=58 \%)$ a $370^{\circ} \mathrm{C}$. Dessa forma, o uso dessa amostra é duplamente vantajoso do ponto de vista econômico: ela é mais ativa e seletiva e pode ser usada numa temperatura mais baixa $\left(350^{\circ} \mathrm{C}\right)$ diminuindo os custos de energia. Isto permite a operação numa única etapa, eliminando a reação de LTS o que também reduz os custos de produção.

\section{CONCLUSÕES}

$\mathrm{Na}$ faixa de temperatura de $250 \mathrm{a} 400^{\circ} \mathrm{C}$, catalisadores à base de hematita e contendo cobre e alumínio operam sob regime cinético, na reação de HTS, apresentando um aumento da atividade com a temperatura.

A presença do cobre favorece a formação da fase ativa (magnetita) e sua estabilidade. O alumínio atua aumentando a área específica (promotor textural) enquanto o cobre aumenta a atividade dos sítios catalíticos (promotor estrutural). O papel textural do alumínio pode ser atribuído a uma ação na superfície (em que ele separa as partículas de óxido de ferro, evitando a sinterização) e na rede do sólido, onde ele causa tensões, favorecendo a formação de partículas menores. No caso do cobre, essa ação pode ser atribuída a modificações eletrônicas nos átomos de ferro, causados por interações com o cobre.

Os catalisadores obtidos podem operar na faixa de temperatura estudada, sem sofrer perda de atividade. Isto indica que o seu emprego pode reduzir os custos de processo, pela operação a baixas temperaturas e que esses materiais possuem resistência à desativação, refletida pelo seu bom desempenho a altas temperaturas. $\mathrm{O}$ catalisador mais promissor para uma aplicação industrial é aquele contendo os dois metais, que é mais ativo e seletivo que uma amostra comercial à base de cromo e cobre. O sólido obtido possui a vantagem de não ser tóxico, ao contrário do comercial, devido à substituição do cromo pelo alumínio, e poder ser utilizado a temperaturas mais baixas $\left(350{ }^{\circ} \mathrm{C}\right)$, viabilizando a conversão do monóxido de carbono, em uma única etapa.

\section{AGRADECIMENTOS}

Os autores agradecem ao CNPq, FINEP e ao CNPq/PADCT/ CEMAT pelo suporte financeiro. G.C.A. e A.O.S. agradecem ao $\mathrm{CNPq}$ e à CAPES pelas bolsas concedidas. 


\section{REFERÊNCIAS}

1. Campbell, J. S.; Craven, P.; Young, P. W.; Catalyst Handbook, Wolfe Scientific Books: London, 1970, p. 97.

2. Nielsen, A.; Bohlbro, H.; An Investigation on Promoted Iron Catalysts for the Synthesis of Ammonia, Gjellerup Forlag: Copenhagen, 1967, p. 221.

3. Bohlbro , H.; Investigation on the Kinetics of the Conversions of Carbon Monoxide with Water Vapor over Iron Oxide based Catalysts, $2^{\text {nd }}$ ed., The Haldor Topsoe Research Laboratory: Copenhagen, 1960, p. 6.

4. Twigg, M. V.; Lloyd, L.; Ridler, D. E.; Catalyst Handbook, Manson Publishing Ltda: London, 1996, p. 283

5. Newsome, E.; David, S.; Catal. Rev.- Sci. Eng. 1980, 21, 275.

6. Robin, A. M. ; Tassoney, J. P.; US pat. 3,652,454 1972.

7. Tamaru, A.; Yoshikazu, O; Hashimoto, H.; Honda, K.; Ger. Offen. DE 3,835,345 1987.

8. Huang, D.C.; Braden, J. L.; US pat. 4,861,745 1989

9. Souza, M. O. da G.; Quadro, E. B.; Rangel, M. C.; Quim. Nova 1998, 21, 428.

10. Quadro, E. B.; Dias, M. de L. R.; Amorim, A. M. M.; Rangel, M. C.; J. Braz. Chem. Soc. 1999, 10, 51.

11. Souza, A. O.; Santos, T. S. M.; Rangel, M. C.; XV Simpósio Iberoamericano de Catálise, 1996.

12. Araujo, G. C.; Rangel, M. C.; Stud. Surf. Sci. Catal. 2000, 130, 1601.
13. Kokai, J.; JP 60 54,901 1985.

14. Vogel, A.; Denney, R. C.; Jeffery, G. H.; Medham, J.; Análise Inorgânica Quantitativa, Ed. Guanabara Koogan S.A.: Rio de Janeiro, 1984, p. 268.

15. Olweiller, O. A.; Química Analítica Quantitativa, Ed. Livros Técnicos e Científicos S.A.: Rio de Janeiro, 1981.

16. Alexéev, V.; Análise Quantitativa, Ed. Lopes da Silva: Porto, 1984.

17. Kolthoff, I. M.; Moskovitz, B.J.; Anal. Chem. 1937, 41, 629.

18. Sidhu, P. S. ; Gilkes, R. J.; Posner, A. M.; J. Inorg. Nucl. Chem. 1978, 40, 429.

19. Dry, M. E.; Plessis, J. A. K.; Leuritz, G.; J. Catal. 1966, 6, 194.

20. Naono, H.; Fujiwara, R.; J. Colloid Interf. Sci. 1980, 2, 73.

21. Pascal, P.; Nouveau Traité de Chimie Minérale, tome XVII, Masson et Cie: Paris, 1967.

22. Rangel, M.C; Galembeck, F.; J. Catal. 1994, 145, 364

23. Fischer, W. R.; Shwertmann, V.; Clays Clay Miner. 1975, 23, 33.

24. Topsoe, H.; Dumesic, J. A.; Boudart, M.; J. Catal. 1978, 28, 477.

25. Matijevic, E.; Scheiner, P.; J. Colloid Interf. Sci. 1978, 63, 509.

26. Viswanath, R. P.; Viswanath, B.; Sastri, M. V. C.; Thermochim. Acta 1976, $16,240$.

27. Rangel, M. C.; Sassaki, R. M.; Galembeck, F.; Catal. Lett. 1995, 33, 237.

28. Boreskov, J. K.; Yureva, T. M.; Sergeeva, A.S.; Kinet. Katal. 1970, 11,1476

29. Podolski, W. F.; Kim, Y. G.; Ind. Eng. Chem. Process, Des. Dev. 1974, 13, 415 . 